ARTIGO ORIGINAL ORIGINAL ARTICLE

\section{Condutas clínicas e barreiras no tratamento da polineuropatia amiloidótica familiar associada à transtirretina (PAF-TTR) no Brasil}

\author{
Clinical practice and barriers in familial transthyretin- \\ related amyloidotic polyneuropathy (FAP) care in Brazil \\ Ana Carolina Padula Ribeiro Pereira', Roberta Arinelli Fernandes ${ }^{1}$, Ellen Cristina \\ Alves de Paula ${ }^{2}$, Anderson Luiz Machado Freitas ${ }^{3}$, Denise Batista Nunes ${ }^{4}$, Ana Elisa \\ Rodrigues Marangon 5 , Deilys Gonzalez Vazquez ${ }^{5}$, Karyn Regina Jordão Koladicz ${ }^{6}$
}

DOI: $10.21115 / J B E S . v 13 . n 2 . p 128-35$

\section{Palavras-chave:}

amiloidose familiar, neuropatias amiloides familiares, inquéritos e questionários, conduta do tratamento medicamentoso

\section{Keywords:}

familial amyloidosis, familial amyloid neuropathies, surveys and questionnaires, medication therapy management

\section{RESUMO}

Objetivo: Identificar as condutas terapêuticas e a variabilidade na prática clínica, assim como necessidades não atendidas e barreiras para a adequada assistência a pacientes com polineuropatia amiloidótica familiar relacionada à transtirretina (PAF-TTR), no Brasil. Métodos: Estudo transversal, por meio de questionário semiestruturado on-line enviado por e-mail. Foram incluídos médicos com experiência no manejo clínico-assistencial de pacientes com PAF-TTR no Brasil. O questionário foi composto por 30 questões envolvendo características gerais da população brasileira com PAF-TTR, características das escolhas terapêuticas e da falha, definições de progressão de doença e estadiamento, e métodos para mensuração do impacto na qualidade de vida. Resultados: Seis profissionais responderam ao inquérito. Quanto ao diagnóstico e à classificação da doença, houve consenso quanto ao uso de quadro clínico associado a testagem genética para o diagnóstico, e foram considerados adequados os critérios de Coutinho e do Ministério da Saúde, apesar de serem pouco úteis na avaliação da progressão da doença. Entre os especialistas, 83,3\% entendem que a terapia atualmente disponível no Sistema Único de Saúde (SUS) atende às necessidades dos pacientes no estágio I da doença, entretanto todos os especialistas apontam necessidades assistenciais não atendidas, uma vez que esse medicamento não possui benefício definido para os estágios II e III da doença. A progressão da doença é definida como qualquer novo sintoma ou piora daqueles preexistentes, não sendo necessária modificação no estágio da doença para caracterizar tal evento. Conclusões: A condução deste estudo permitiu a identificação de aspectos importantes para auxiliar no entendimento da prática clínico-assistencial no país e das necessidades em saúde desses pacientes.

\begin{abstract}
Objective: To identify therapeutic approaches and variability in clinical practice, as well as unmet needs and barriers to adequate care for patients with familial transthyretin-related amyloidotic polyneuropathy (FAP) in Brazil. Methods: Cross-sectional study using an online semi-structured questionnaire sent by email. Physicians with experience in the clinical-care management of patients with FAP in Brazil were included. The questionnaire consisted of 30 questions involving general characteristics of the Brazilian population with FAP; characteristics of therapeutic choices and failure; definitions of disease progression and staging; and methods for measuring the impact on quality of life. Results: Six professionals responded to the survey. As for the diagnosis and classification of
\end{abstract}

\footnotetext{
Recebido em: 30/06/2021. Aprovado para publicação em: 03/08/2021.

1. Origin Health Company, São Paulo, SP, Brasil.

2. Gerente de Acesso ao Mercado, PTC Farmacêutica do Brasil, São Paulo, SP, Brasil.

3. Diretor de Acesso ao Mercado, PTC Farmacêutica do Brasil, Brasília, DF, Brasil.

4. Diretora associada de Assuntos Médicos, PTC Farmacêutica do Brasil, São Paulo, SP, Brasil.

5. Gerente de Assuntos Médicos, PTC Farmacêutica do Brasil, São Paulo, SP, Brasil.

6. Diretora executiva de Assuntos Médicos, PTC Farmacêutica do Brasil, SC, Brasil.

Instituição onde o trabalho foi executado: ORIGIN Health Company

Congresso: $O$ presente estudo não foi apresentado em nenhum congresso.

Financiamento: Este estudo teve apoio financeiro da PTC Farmacêutica do Brasil Ltda. para sua realização.

Autor correspondente: Ellen Cristina Alves de Paula. Rua Castilho, 392, 170 andar, Brooklin, São Paulo, SP, Brasil. CEP: 04568-010.
}

Telefone: (11) 99477-3969. E-mail: epaula@ptcbio.com 
the disease, there was a consensus regarding the use of a clinical picture associated with genetic testing for the diagnosis and, as appropriate, the criteria proposed by Coutinho and the Ministry of Health, although not very useful in evaluating the progression of the disease. $83.3 \%$ of experts understand that the therapy currently available in the SUS meets the needs of patients in stage I of the disease, however, all experts point out unmet care needs, since this drug has no defined benefit for stages II and III of the disease. Disease progression is defined as any new symptom or worsening of pre-existing ones, and no change in the stage of the disease is necessary to characterize such an event. Conclusions: The conduction of this study allowed to identify important aspects to a better understanding of the clinical care practice in the country and unmet needs of these patients.

\section{Introdução}

A amiloidose hereditária associada à transtirretina, também conhecida como polineuropatia amiloidótica familiar relacionada à transtirretina (PAF-TTR), é uma doença rara, genética, de herança autossômica dominante, causada por mutações no gene TTR. Essas mutações levam à produção da proteína TTR em conformação instável, formando fibras amiloides que se depositam em diversos tecidos e órgãos, sendo a PAF-TTR uma doença multissistêmica e progressiva. Dentre as principais manifestações, destacam-se o declínio na capacidade funcional e motora, as disfunções cardiovasculares, gastrointestinais, renais e oculares, e o grande impacto na autonomia e na qualidade de vida de pacientes e cuidadores (Adams et al., 2016; Adams et al., 2021). Além disso, é uma doença fatal - quando não tratada, a sobrevida média desde o início da doença varia de 6 a 12 anos (Coelho et al., 2013).

De acordo com as manifestações apresentadas, os pacientes com PAF-TTR podem ser classificados em diferentes estágios da doença (Pinto et al., 2018). Uma classificação amplamente utilizada é a de Coutinho e colaboradores, que categoriza a doença em três diferentes estágios, de acordo com os seguintes critérios: (i) estágio l: neuropatia sensorial e motora limitada aos membros inferiores, dor e sensibilidade térmica gravemente prejudicadas, com sensibilidade ao toque leve e propriocepção relativamente poupadas (dissociação sensorial), comprometimento motor leve e deambulação sem a necessidade de dispositivos de auxílio à locomoção; (ii) estágio II: necessidade de dispositivos de auxílio à locomoção (andadores ou bengalas), neuropatia atingindo membros superiores e tronco, amiotrofia em membros inferiores e superiores e comprometimento motor moderado; e (iii) estágio III: estágio terminal, acamado ou em uso de cadeira de rodas, neuropatia sensorial, motora e autonômica grave, afetando todos os membros. No Protocolo Clínico e Diretrizes Terapêuticas (PCDT) da Polineuropatia Amiloidótica Familiar (Brasil, 2018b), documento do Ministério da Saúde do Brasil, a classificação utilizada é a do Consenso Europeu, que se baseou no estadiamento de Coutinho e no escore PND (Polyneuropathy Disability Score), de acordo com as seguintes características: (i) estágio 0: assintomático; (ii) estágio I/inicial: sintomatologia leve, ambulatorial, limitada aos membros inferiores, e escores PND I (distúrbios sensitivos nas extremidades, contudo a capacidade de deambulação encontra-se preservada) e
Il (dificuldade para deambulação, porém sem a necessidade de bengala); (iii) estágio I//intermediário: sintomas moderados, deterioração neuropática adicional, ambulatorial, mas requer assistência, escores PND IIla (necessária uma bengala ou muleta para deambulação) e Illb (necessárias duas bengalas ou duas muletas para deambulação); (iv) estágio III/avançado: sintomatologia grave, acamado/cadeira de rodas, com fraqueza generalizada, e escore PND IV (paciente confinado à cadeira de rodas ou cama). O estadiamento da doença é importante para avaliar o prognóstico dos pacientes e guiar a escolha do manejo mais adequado (Adams et al., 2016; Pinto et al., 2018; Plante-Bordeneuve, 2018).

O transplante hepático foi o primeiro tratamento proposto para pacientes em estágios iniciais da PAF-TTR, sendo associado com aumento da expectativa de vida em comparação à história natural da doença (Ericzon et al., 2015). Em relação ao tratamento farmacológico, importantes avanços ocorreram nas últimas décadas. O primeiro medicamento desenvolvido foi o tafamidis, um estabilizador da proteína TTR indicado para pacientes em estágio I da doença sem transplante prévio. Entretanto, não há dados para suportar o uso desse medicamento no tratamento de pacientes em estágio mais avançado da doença (Adams et al., 2016; Pinto et al., 2018; Brasil, 2018a). Mais recentemente, foram desenvolvidos os silenciadores de mRNA inotersena e patisirana, que bloqueiam a tradução da proteína TTR e são indicados para o tratamento de pacientes em estágios I e II ou sem resposta ao tafamidis (Benson et al., 2018; Adams et al., 2018).

Atualmente, o único medicamento aprovado para comercialização no Brasil é o tafamidis. Inotersena e patisirana são registradas pela Agência Nacional de Vigilância Sanitária (Anvisa), porém ainda não estão disponíveis para comercialização. O tafamidis também está incorporado no Sistema Único de Saúde (SUS), sendo recomendado para o tratamento de pacientes com PAF-TTR em estágio I sem transplante hepático prévio (Brasil, 2018a). Pacientes em estágios mais avançados da doença e pacientes que não respondem ao tratamento com tafamidis não possuem tratamento farmacológico disponível no Brasil. Esse fato evidencia importantes necessidades médicas não atendidas para pacientes com PAF-TTR no Brasil.

Informações sobre as práticas clínico-assistenciais no manejo de pacientes com PAF-TTR no país são escassas. A avaliação da prática clínica por meio da percepção de especialistas 
e da contextualização de cenários é um passo importante no processo de avaliação de novas tecnologias em saúde, uma vez que pode apontar necessidades relacionadas ao acesso do paciente ao tratamento em questão, tornando-se parte importante na etapa inicial do processo de incorporação nos sistemas de saúde. Dessa forma, considerando a escassez de informações na literatura atual, os recentes avanços e os desafios no cuidado desses pacientes, o objetivo do presente estudo foi identificar as condutas terapêuticas e a variabilidade na prática clínica, assim como necessidades não atendidas e barreiras para a adequada assistência a esses pacientes no contexto nacional.

\section{Métodos}

\section{Delineamento}

Estudo transversal identificando a prática clínico-assistencial de médicos que tratam pacientes com PAF-TTR por meio de inquérito on-line, conduzido entre outubro de 2020 e fevereiro de 2021.

\section{Amostra/critérios de inclusão}

Participantes elegíveis consistiam em médicos com experiência no manejo clínico-assistencial de pacientes com PAF-TTR no Brasil, seja em serviços públicos e/ou privados.
Os profissionais foram identificados a partir de publicações na literatura científica, comunicados de imprensa e contato com líderes de opinião. Esses profissionais foram contatados por telefone e correio eletrônico para a confirmação de critérios de elegibilidade. Se elegíveis, questionários semiestruturados foram enviados por e-mail, com o prazo-limite de duas semanas para resposta. Os participantes não foram informados sobre a fonte de financiamento do estudo, e o patrocinador não teve envolvimento no processo de identificação e recrutamento deles.

\section{Ética}

Todos os respondedores consentiram em participar do estudo. Considerando que não foram coletados dados individualizados de pacientes, apenas a opinião dos profissionais e suas percepções com sua casuística, não foi necessária a aprovação do projeto por Comitê de Ética em Pesquisa.

\section{Descrição do instrumento}

O questionário foi composto por 30 questões, estruturadas nas seguintes seções: características gerais da população brasileira com PAF-TTR; características das escolhas terapêuticas e da falha; definições de progressão de doença e estadiamento; e métodos para mensuração do impacto na qualidade de vida. As questões são apresentadas no quadro 1.

Quadro 1. Estrutura do questionário aplicado no estudo

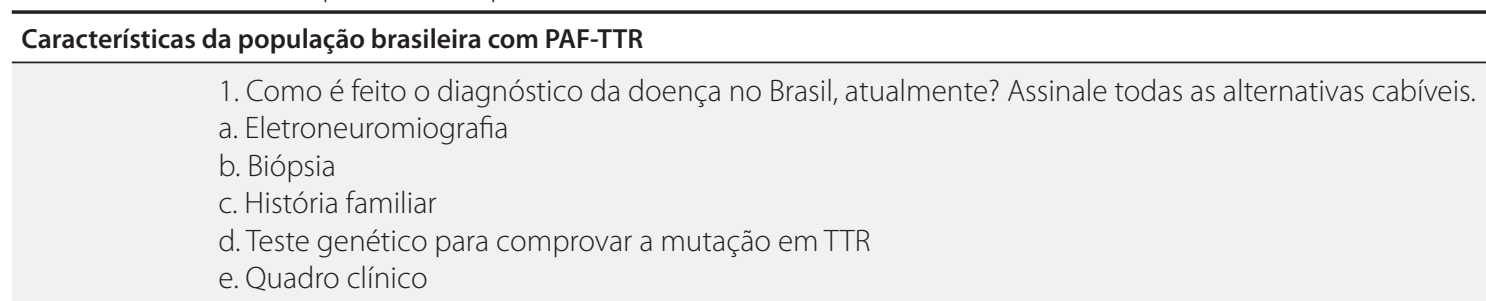

2. Você costuma acompanhar os seus pacientes com PAF-TTR em conjunto com outras especialidades (atendimento multidisciplinar)? 2.1 Se sim, como funciona essa conduta multidisciplinar?

3. Qual a idade média ao diagnóstico dos pacientes com PAF-TTR no Brasil?

4. Segundo o Protocolo Clínico e Diretrizes Terapêuticas (PCDT) da PAF, proposto pelo Ministério da Saúde em 2018, a doença pode ser classificada de acordo com os critérios expostos no PCDT. Você concorda com esses critérios? Se não concorda, qual seria o critério ideal na sua opinião?

5. O que você entende como estágio inicial e intermediário da doença?

6. Como se dividem os pacientes, de acordo com a classificação proposta pelo PCDT, na sua prática clínica? (informar em porcentagem).

7. Ainda sobre a classificação da doença, Pinto e colaboradores (2018), no Consenso Brasileiro para o diagnóstico, manejo e tratamento da PAF-TTR, recomendam a utilização dos critérios propostos por Coutinho. Você concorda com esses critérios? Se não concorda, qual seria o critério ideal na sua opinião?

8. Como se dividem os pacientes, de acordo com a classificação proposta pelo Consenso Brasileiro, na sua prática clínica?

9. Insira abaixo quaisquer outras informações que julgue necessárias em relação a diferentes características da população brasileira com diagnóstico de PAF-TTR.

\section{Características das escolhas terapêuticas e da falha}

10. Atualmente, como o paciente com PAF-TTR é tratado, considerando as diferentes classificações da doença baseado no PCDT, no contexto da saúde pública (SUS)?

11. Atualmente, como o paciente com PAF-TTR é tratado, considerando as diferentes classificações da doença, no contexto da saúde privada? 


\section{Características da população brasileira com PAF-TTR}

12. Sendo o tafamidis o único medicamento incorporado no SUS, você entende que ainda existem perfis de pacientes que não são atendidos por essa opção terapêutica? Se sim, quais seriam esses perfis?

13. Para qual perfil de pacientes você indicaria um silenciador como primeira opção terapêutica? Responda com base na sua prática clínica.

14. Como você avaliaria a eficácia relativa de um estabilizador versus silenciador gênico no tratamento de pacientes em estágio II de Coutinho?

15. Você considera que a terapia atualmente contemplada no PCDT atende em plenitude a todos os perfis de pacientes classificados como estágio I de Coutinho?

16. Você considera que os dados de eficácia publicados de tafamidis são suficientes para a tomada de decisão por essa terapia para pacientes em estágio II (Coutinho)?

17. O que, na sua opinião, caracteriza falha terapêutica ao tafamidis e em que momento você consideraria a troca para um silenciador? Abaixo foram incluídos parâmetros descritos no PCDT em que são esperados benefícios com o uso do tafamidis. Descreva como você classificaria a falha em cada critério e em que momento a troca para o silenciador seria necessária.

18. O que você considera como definição da falha terapêutica de um estabilizador? Quando você consideraria a troca para um silenciador?

19. Em uma escala de 0-100\%, como você classificaria sua abertura para uma nova estratégia terapêutica para o manejo de pacientes com PAF-TTR no estágio I com falha terapêutica a tafamidis e estágio II no Brasil?

20. Insira abaixo quaisquer outras informações que julgue necessárias em relação às escolhas terapêuticas e falha na população brasileira com diagnóstico de PAF-TTR.

\section{Definições de progressão de doença e estadiamento}

21. O que você considera como progressão da doença?

22. Como você avalia a progressão da doença na sua prática clínica? Assinale todas as alternativas cabíveis.

23. No seu entendimento, as escalas de estadiamento hoje utilizadas (Coutinho e PND) avaliam adequadamente todos os possíveis acometimentos neurológicos causados pela doença?

24. Você entende que há necessidade de uma diretriz que defina as características da progressão da doença em pacientes com diagnóstico de PAF-TTR? Justifique.

25. Insira abaixo quaisquer outras informações que julgue necessárias em relação a definições de progressão da doença e estadiamento da população brasileira com diagnóstico de PAF-TTR.

\section{Métodos para mensuração do impacto na qualidade de vida}

26. O que caracteriza o impacto da PAF-TTR na qualidade de vida do paciente?

27. Como você mensura o impacto da doença na qualidade de vida dos pacientes, na sua prática clínica? Se utiliza alguma escala para validação, mencione-a.

28. Existem outras ferramentas de avaliação de qualidade de vida que você não utiliza na prática clínica, mas que você considera relevantes e mensuráveis? Assinale todas as alternativas cabíveis.

29. Você considera a melhora na qualidade de vida como um dos objetivos primários no tratamento de PAF-TTR no Brasil? Justifique.

30. De acordo com o seu conhecimento, você considera que a melhora na qualidade de vida dos pacientes pode ser entendida como um desfecho de eficácia do tratamento? Justifique.

\section{Análise dos dados}

Foi realizada análise quantitativa dos resultados por meio de estatísticas descritivas, como frequências, proporções, mediana e amplitude. Além disso, foi realizada análise qualitativa dos comentários realizados. O patrocinador não esteve envolvido com a análise dos dados.

\section{Resultados}

Quinze profissionais foram convidados para o presente estudo, dos quais seis responderam ao inquérito. Os motivos de não participação foram relacionados à inviabilidade de tempo ou por percepção de conflito de interesse decorrente da sua participação em estudos clínicos, mesmo esse não sendo um fator excludente. O perfil dos respondedores e dos não respondedores está apresentado na tabela 1.

\section{Processo diagnóstico e classificação da doença}

A tabela 2 sumariza as percepções dos profissionais em relação ao diagnóstico e à classificação dos pacientes com PAF-TTR no Brasil.

Houve consenso entre os especialistas no uso de quadro clínico associado a testagem genética para o diagnóstico de PAF-TTR no Brasil; há variabilidade no entendimento sobre o uso de biópsia de nervo, que pode ter utilidade em casos duvidosos, para diagnóstico diferencial com outras polineu- 
ropatias e de exames auxiliares inespecíficos, tal como a eletroneuromiografia. Há a percepção de redução do uso de biópsia devido ao maior acesso a exames genéticos.

Os participantes entendem como adequados os critérios propostos por Coutinho e pelo Ministério da Saúde, com maior aceitabilidade para os critérios apresentados pelo Ministério da Saúde. Contudo, ressaltam que esses estágios são pouco úteis na avaliação da progressão da doença, uma vez que a mudança de estágio só ocorre após grande acúmulo de perdas. As definições apresentadas pelos especialistas

Tabela 1. Principais características dos respondedores e não respondedores

\begin{tabular}{lcc}
\hline & Respondedores $(\mathbf{n}=\mathbf{6})$ & Não respondedores $(\mathbf{n}=\mathbf{9})$ \\
\cline { 2 - 3 } & $\mathbf{N}(\%)$ & $\mathbf{N}(\%)$ \\
\hline Sexo feminino & $1(16,7)$ & $(33,3)$ \\
\hline Região & & 0 \\
\hline Centro-oeste & 5 & 3 \\
\hline Sudeste & 0 & 2 \\
\hline Sul & 1 & 3 \\
\hline Nordeste & 0 & 1 \\
\hline Norte & & \\
\hline Atuação & $6(100)$ & $9(100)$ \\
\hline Saúde pública (Sistema Único de Saúde - SUS) & $4(66,6)$ & $5(55,6)$ \\
\hline Saúde suplementar/prática privada & & \\
\hline Especialidade & $5(83,3)$ & $9(100)$ \\
\hline Neurologia & 0 & 0 \\
\hline Genética médica & $1(16,6)$ & 0 \\
\hline Outras & & \\
\hline
\end{tabular}

Tabela 2. Percepções dos profissionais sobre processo diagnóstico e classificação de PAF-TTR no Brasil

\begin{tabular}{|c|c|}
\hline & N (\%) ou mediana (amplitude) \\
\hline \multicolumn{2}{|l|}{ Recursos utilizados para diagnóstico de PAF-TTR no Brasil } \\
\hline Quadro clínico & $6(100)$ \\
\hline Testagem genética & $6(100)$ \\
\hline História familiar & $5(83,3)$ \\
\hline Biópsia de nervo & $4(66,7)$ \\
\hline Eletroneuromiografia & $1(16,7)$ \\
\hline \multicolumn{2}{|l|}{ Profissionais que recomendam a utilização dos estágios clínicos de PAF-TTR } \\
\hline Conforme Coutinho & $5(83,3)$ \\
\hline Conforme PCDT da Polineuropatia Amiloidótica Familiar do Ministério da Saúde & $6(100)$ \\
\hline \multicolumn{2}{|c|}{$\begin{array}{l}\text { Proporção de pacientes de acordo com apresentação clínica, conforme estágios apresentados } \\
\text { no PCDT da Polineuropatia Amiloidótica Familiar do Ministério da Saúde }\end{array}$} \\
\hline Estágio 0 & $30(15-30)$ \\
\hline Estágio I & $35(20-50)$ \\
\hline Estágio II & $30(10-3)$ \\
\hline Estágio III & $10(10-15)$ \\
\hline \multicolumn{2}{|c|}{$\begin{array}{l}\text { Proporção de pacientes de acordo com apresentação clínica, conforme estágios propostos por } \\
\text { Coutinho }\end{array}$} \\
\hline Estágio I & $50(25-70)$ \\
\hline Estágio II & $30(15-40)$ \\
\hline Estágio III & $12,5(10-20)$ \\
\hline
\end{tabular}


para estágios inicial e intermediário da doença estão em linha com as definições de estágio I e II, respectivamente, tanto conforme Coutinho quanto conforme o Ministério da Saúde.

Os especialistas relatam observar o diagnóstico sendo realizado majoritariamente em pacientes entre 30 e 40 anos de idade, com segundo pico de incidência em pacientes na sexta década de vida. Por fim, os especialistas salientam a inexistência de estudos populacionais amplos no Brasil sobre PAF-TTR e o desconhecimento da comunidade médica a respeito da condição, impactando em atraso diagnóstico.

\section{Abordagem terapêutica}

Todos os respondentes sinalizaram que na sua prática é realizado atendimento por equipe multidisciplinar. Contudo, na composição da equipe apenas foi sinalizado o envolvimento de outras especialidades médicas, como cardiologia, gastroenterologia, hematologia, nefrologia e oftalmologia, não sendo mencionadas áreas da saúde não médicas.

Sobre o tratamento farmacológico, o tafamidis é referido por todos os especialistas como alternativa terapêutica para o estágio I da doença. O tafamidis também é referido como alternativa terapêutica no estágio II da doença, tanto na saúde pública como suplementar, por 66,7\% dos especialistas. Desses, dois especialistas referiram acesso a essa tecnologia por via judicial, uma vez que não está incorporado no SUS ou na saúde suplementar. Os silenciadores de mRNA (inotersena e patisirana), apesar de não possuírem autorização para comercialização no Brasil no momento do inquérito, foram sinalizados como alternativa terapêutica no estágio \|l da doença por 50\% dos especialistas na saúde suplementar e por apenas um (16,7\%) especialista no sistema público. Além disso, um especialista $(16,7 \%)$ considerou-os como alternativa para os estágios I e III na saúde suplementar. O transplante hepático parece ter uso bastante limitado, seja no SUS, seja na saúde suplementar (Tabela 3).

A maioria dos especialistas (83,3\%) entendem que a terapia atualmente disponível no SUS atende às necessidades dos pacientes no estágio I da doença, salientando que o tafamidis pode ser utilizado nos tratamentos iniciais. Entretanto, todos os especialistas entendem que, com o tafamidis sendo o único medicamento incorporado no SUS, há necessidades assistenciais não atendidas, uma vez que esse medicamento não possui benefício definido para os estágios II e III da doença e que há pacientes que progridem na vigência de seu uso. Para o tratamento de pacientes com PAF-TTR em estágio II, quatro $(66,7 \%)$ especialistas possuem a percepção de que os silenciadores de mRNA possuem eficácia superior à do tafamidis, enquanto um $(16,7 \%)$ considera que eles possuem efetividade semelhante e um (16,7\%) não possui opinião formada; todavia, os especialistas salientam a inexistência de estudos clínicos avaliando essa comparação. Os especialistas sinalizaram potencial uso preferencial dos silenciadores de mRNA em pacientes em estágio || e estágio I tardio, pacientes em deterioração clínica em vigência de uso de tafamidis e em pacientes com rápida evolução clínica. Os seguintes critérios foram mencionados como importantes para considerar falha terapêutica com o tafamidis e início de terapia com silenciadores de mRNA: a progressão da neuropatia periférica, o surgimento ou a piora de sintomas desautonômicos e a piora do status nutricional.

Tabela 3. Prática terapêutica de acordo com estágio da doença, no sistema público e na saúde suplementar

\begin{tabular}{|c|c|c|c|}
\hline \multirow{2}{*}{ Estágio } & \multirow{2}{*}{ Tratamento } & Sistema público & Saúde suplementar \\
\hline & & $N(\%)$ & $\mathrm{N}(\%)$ \\
\hline \multirow[t]{4}{*}{0} & Sem tratamento farmacológico específico & $6(100)$ & $6(100)$ \\
\hline & Estabilizador de TTR (tafamidis) & 0 & 0 \\
\hline & Silenciadores de mRNA (inotersena ou patisirana) & 0 & 0 \\
\hline & Transplante hepático & 0 & 0 \\
\hline \multirow[t]{4}{*}{ | } & Sem tratamento farmacológico específico & 0 & 0 \\
\hline & Estabilizador de TTR (tafamidis) & $6(100)$ & $6(100)$ \\
\hline & Silenciadores de mRNA (inotersena ou patisirana) & 0 & $1(16,3)$ \\
\hline & Transplante hepático & $1(16,3)$ & $1(16,3)$ \\
\hline \multirow[t]{4}{*}{$\|$} & Sem tratamento farmacológico específico & 0 & 0 \\
\hline & Estabilizador de TTR (tafamidis) & $4(66,7)$ & $4(66,7)$ \\
\hline & Silenciadores de mRNA (inotersena ou patisirana) & $1(16,3)$ & $3(50)$ \\
\hline & Transplante hepático & $2(33,3)$ & $1(16,3)$ \\
\hline \multirow[t]{4}{*}{ III } & Sem tratamento farmacológico específico & $6(100)$ & $5(83,3)$ \\
\hline & Estabilizador de TTR (tafamidis) & 0 & 0 \\
\hline & Silenciadores de mRNA (inotersena ou patisirana) & 0 & $1(16,7)$ \\
\hline & Transplante hepático & 0 & 0 \\
\hline
\end{tabular}




\section{Acompanhamento clínico}

Todos os especialistas entendem progressão da doença como sendo qualquer novo sintoma ou piora de sintomas preexistentes, não sendo necessária modificação no estágio da doença para caracterizar progressão. Na prática clínica, os participantes relataram avaliar a progressão da doença principalmente pela capacidade de locomoção (83,3\%), pelos distúrbios sensitivos $(83,3 \%$ ) e pelos distúrbios autonômicos (83,3\%). Outros aspectos incluem a avaliação do estado nutricional $(16,6 \%)$, questionários de qualidade de vida $(16,6 \%)$, piora dos parâmetros analisados por testes como o SUDOSCAN, CHEPS, OST $(16,6 \%)$ e avaliação por exames complementares, como eletroneuromiografia, biópsia tecidual, eletrocardiograma, ecocardiograma, cintilografia, função renal, entre outros $(16,6 \%)$.

Todos os especialistas consideraram que melhoria na qualidade de vida é um objetivo primário no tratamento de PAF-TTR no Brasil, com cinco deles (83,3\%) considerando como um desfecho de eficácia terapêutica. Entre os instrumentos relevantes para a avaliação, foram citados o mNIS+7, a Norfolk QOL-DN e o SF-36.

Quatro $(66,7 \%)$ dos especialistas entendem que há necessidade de uma diretriz que defina as características da progressão da doença em pacientes com diagnóstico de PAF-TTR. Características mencionadas para essa diretriz foram: ser sensível, definir um protocolo mínimo de seguimento, definir falha terapêutica a partir de critérios objetivos e contemplar os aspectos multissistêmicos da doença.

\section{Discussão}

A PAF-TTR é uma doença crônica, multissistêmica e progressiva, possuindo importante impacto em morbimortalidade e na qualidade de vida de pacientes e seus cuidadores (Adams et al., 2016; Adams et al., 2021; Coelho et al., 2013). Nesse inquérito, realizado com seis especialistas em PAF-TTR com atuação no SUS e na saúde suplementar no Brasil, foram identificados aspectos importantes para auxiliar no entendimento da prática clínico-assistencial no país e das necessidades em saúde desses pacientes.

Alguns avanços relacionados à prática assistencial foram identificados. O maior acesso à testagem genética favorece a identificação de casos novos, reduzindo também a necessidade de testes mais invasivos, como biópsias de nervo. Documentos recentes, como o PCDT da Polineuropatia Amiloidótica Familiar do Ministério da Saúde e o Consenso Brasileiro para o Diagnóstico, Manejo e Tratamento da Polineuropatia Amiloidótica Familiar associada à Transtirretina, ambos publicados em 2018, auxiliam na disseminação de informações sobre a doença para os profissionais de saúde, assim como reduzem a variabilidade na prática clínica (Pinto et al., 2018; Brasil, 2018b). Além disso, recentemente, outros documentos, frutos de colaborações internacionais, vêm sendo desenvolvidos com o mesmo propósito (Adams et al., 2021).
Contudo, ainda se observa grande parcela dos pacientes sendo diagnosticados em estágios mais avançados da doença: conforme relatado em nosso inquérito, cerca de metade dos pacientes apresenta doença em estágios intermediários ou avançados, no qual a abordagem farmacológica disponível é menos eficiente (Brasil, 2018b; Benson et al., 2018; Adams et al., 2018; Coelho et al., 2012).

Sobre a prática prescricional, identificamos alguns aspectos interessantes. Apesar da ausência de estudos avaliando o uso do tafamidis em pacientes com estágio II da doença, $66,7 \%$ dos especialistas referem utilizar esse medicamento na sua prática clínica para esses pacientes, provavelmente por esse ser o único medicamento atualmente disponível com indicação para uso em PAF-TTR. Digno de nota, o principal acesso a esse tratamento é dado por via judicial, uma vez que a Comissão Nacional de Incorporação de Tecnologias em Saúde (Conitec) aprovou o uso no SUS exclusivamente para pacientes em estágio I, explicitando a ausência de evidência de benefício em pacientes em estágios intermediários ou avançados (Brasil, 2018a). É compreensível, do ponto de vista clínico, o uso compassivo de medicamentos sem evidência na ausência de alternativas terapêuticas, mas deve-se considerar que essa prática está associada a custos e riscos de eventos adversos, sem a certeza de benefício para o paciente.

No Brasil, a abordagem terapêutica, até então limitada ao uso do tafamidis e ao transplante hepático em situações pontuais, passa a contar com uma nova classe de medicamentos: os silenciadores de mRNA. Inotersena e patisirana receberam recentemente a aprovação regulatória da Anvisa e a aprovação para comercialização está prevista para o segundo semestre de 2021. Os silenciadores de mRNA mostraram-se efetivos no tratamento de pacientes em estágio II da doença, que apresentou, inclusive, regressão de sintomas (Benson et al., 2018; Adams et al., 2018). Além disso, esses medicamentos mostraram-se associados a ganhos na qualidade de vida, algo não evidenciado nos estudos avaliando o tafamidis em PAF-TTR (Coelho et al., 2012). Entendemos que os silenciadores de mRNA passarão naturalmente a ser utilizados no tratamento de pacientes com PAF-TTR no estágio II, assim como atenderão a outras necessidades, como o tratamento de pacientes não respondedores ao tafamidis. Todavia, permanecerá, como sinalizado pelos especialistas, a ausência de alternativas efetivas para o manejo de pacientes em estágio mais avançado (estágio III) de PAF-TTR.

Outro ponto importante é a diferente prática prescricional observada no SUS e na saúde suplementar. Dois especialistas (33,3\%) consideraram o uso dos silenciadores de mRNA apenas na prática privada, não considerando seu uso no SUS. Entendemos que há uma diferença de acesso entre esses sistemas, em especial devido ao diferente financiamento desses dois modelos; contudo, é importante atentar para potenciais inequidades e desenvolver alternativas que permitam adequada assistência da população. 
Apesar de os atuais sistemas de classificação da doença seja o proposto por Coutinho, seja o proposto pelo consenso europeu, adotado pelo Ministério da Saúde - terem alta aceitabilidade e auxiliarem na definição terapêutica inicial, eles possuem limitado uso no acompanhamento dos pacientes devido à sua baixa sensibilidade, não devendo ser utilizados isoladamente para a definição de progressão da doença e/ou falha terapêutica. Foi identificada elevada variabilidade no acompanhamento desses pacientes e nas métricas utilizadas para avaliar resposta terapêutica e progressão. A inexistência de parâmetros objetivos dificulta o manejo terapêutico, uma vez que não proporciona padronização para decisões quanto à interrupção ou troca de terapias. É digno de nota que todos os especialistas consideraram importante a utilização de métricas relacionadas à qualidade de vida, em linha com a busca por desfechos importantes para os pacientes.

Como limitações, é importante salientar o caráter exploratório do presente inquérito, com a aplicação de questionário não validado e obtenção de taxa de resposta de apenas $40 \%$ em relação aos profissionais convidados. Todavia, tendo em vista a ausência de outros dados referentes à prática de vida real no Brasil, a característica ultrarrara da doença e a modificação na dinâmica assistencial devido ao surgimento de nova classe terapêutica, o presente estudo proporciona informações interessantes para o entendimento da prática clínico-assistencial no cenário nacional, destacando as necessidades não atendidas e as barreiras para adequada assistência à população.

\section{Conclusões}

Apesar de avanços diagnósticos recentes, com maior acesso à testagem genética e publicação de diretrizes clínicas, ainda se observa grande parcela de pacientes com diagnóstico tardio da doença. Identificamos também várias necessidades terapêuticas não atendidas para a população com PAF-TTR no Brasil, com o tafamidis sendo a única alternativa terapêutica no momento, também sendo utilizado no tratamento para o estágio II, a despeito da inexistência de evidências científicas. Espera-se que os silenciadores de mRNA venham a ocupar a lacuna assistencial em pacientes em estágio II da doença e em pacientes não respondedores ao tafamidis, permanecendo a ausência de terapia específica para pacientes em estágio avançado. A adoção dessas tecnologias tenderá a ser mais rápida na saúde suplementar do que no SUS, poden- do resultar em inequidades. A existência de diferentes estratégias terapêuticas gera a necessidade de parâmetros para avaliar a progressão clínica e a reposta ao tratamento, para os quais, atualmente, não há uma padronização. Há necessidade de definição de métricas para avaliação de progressão e resposta, devendo-se levar em consideração aspectos multissistêmicos da doença, como neuropatia periférica, sintomas desautonômicos, estado nutricional e qualidade de vida.

\section{Referências bibliográficas}

Adams D, Ando Y, Beirão JM, Coelho T, Gertz MA, Gillmore JD, et al. Expert consensus recommendations to improve diagnosis of ATTR amyloidosis with polyneuropathy. J Neurol. 2021;268(6):2109-22.

Adams D, Gonzalez-Duarte A, O'Riordan WD, Yang CC, Ueda M, Kristen AV, et al. Patisiran, an RNAi Therapeutic, for Hereditary Transthyretin Amyloidosis. N Engl J Med. 2018;379(1):11-21.

Adams D, Suhr OB, Hund E, Obici L, Tournev I, Campistol JM, et al.; European Network for TTR-FAP (ATTReuNET). First European consensus for diagnosis, management, and treatment of transthyretin familial amyloid polyneuropathy. Curr Opin Neurol. 2016;29 Suppl 1(Suppl 1):S14-26.

Benson MD, Waddington-Cruz M, Berk JL, Polydefkis M, Dyck PJ, Wang AK, et al. Inotersen Treatment for Patients with Hereditary Transthyretin Amyloidosis. N Engl J Med. 2018;379(1):22-31.

Brasil. Ministério da Saúde. Comissão Nacional de Incorporação de Tecnologias no SUS (Conitec). Relatório de recomendação: Tafamidis meglumina no tratamento da polineuropatia amiloidótica familiar relacionada à proteína transtirretina. Brasília: Ministério da Saúde; 2018a. 43p.

Brasil. Ministério da Saúde. Portaria Conjunta no 22, de 2 de outubro de 2018. Aprova o Protocolo Clínico e Diretrizes Terapêuticas da Polineuropatia Amiloidótica Familiar. Brasília: Ministério da Saúde; 2018b. 26p.

Coelho T, Maia LF, Martins da Silva A, Waddington Cruz M, PlantéBordeneuve V, Lozeron P, et al. Tafamidis for transthyretin familial amyloid polyneuropathy: a randomized, controlled trial. Neurology. 2012;79(8):785-92.

Coelho T, Maurer MS, Suhr OB. THAOS - The Transthyretin Amyloidosis Outcomes Survey: initial report on clinical manifestations in patients with hereditary and wild-type transthyretin amyloidosis. Curr Med Res Opin. 2013;29(1):63-76.

Ericzon BG, Wilczek HE, Larsson M, Wijayatunga P, Stangou A, Pena JR, et al. Liver Transplantation for Hereditary Transthyretin Amyloidosis: After 20 Years Still the Best Therapeutic Alternative? Transplantation. 2015;99(9):1847-54.

Pinto MV, Barreira AA, Bulle AS, Freitas MRG, França MC Jr, Gondim FAA, et al. Brazilian consensus for diagnosis, management and treatment of transthyretin familial amyloid polyneuropathy. Arq Neuropsiquiatr. 2018;76(9):609-21.

Plante-Bordeneuve V. Transthyretin familial amyloid polyneuropathy: an update. J Neurol. 2018;265(4):976-83 